\title{
Structural and luminescence characteristics of cycled submonolayer InAs/GaAs quantum dots with room-temperature emission at $1.3 \mu \mathrm{m}$
}

\author{
S. Krishna, D. Zhu, J. Xu, K. K. Linder, O. Qasaimeh, and P. Bhattacharyaa) \\ Solid State Electronics Laboratory, Department of Electrical Engineering and Computer Science, \\ University of Michigan, Ann Arbor, Michigan 48109-2122 \\ D. L. Huffaker \\ Microelectronics Research Center, Department of Electrical and Computer Engineering, \\ The University of Texas at Austin, Texas 78712-1084
}

(Received 12 July 1999; accepted for publication 1 September 1999)

\begin{abstract}
Quantum dots were grown by molecular beam epitaxy on GaAs substrates using a cycled submonolayer InAs/GaAs deposition technique. Their structural and luminescence characteristics have been compared with conventional self-organized dots. The room-temperature luminescence spectra are characterized by a ground state transition at $1.3 \mu \mathrm{m}$ and additional transitions corresponding to excited states. Cross-sectional transmission electron microscopy indicates that no dislocations are formed if the total InAs thickness is less than 5-6 monolayers. Temperature dependence of the photoluminescence indicates that both types of quantum dots may have nonradiative defects, caused by segregation and related phenomena. (C) 1999 American Institute of
\end{abstract} Physics. [S0021-8979(99)07923-2]

\section{INTRODUCTION}

While $1.55 \mu \mathrm{m}$ transmission is going to remain the mainstay of long-haul lightwave networks, great interest exists in developing reliable and low-cost sources and detectors for operation at $1.3 \mu \mathrm{m}$. Relatively cheap $1.3 \mu \mathrm{m}$ lasers, based on GaAs substrates, could replace most of the expensive and inefficient InP-based light-emitting diodes currently being used. The two techniques that currently look most promising for $1.3 \mu \mathrm{m}$ emission are InGaAsN/GaAs heterostructures ${ }^{1,2}$ and $\operatorname{In}(\mathrm{Ga})$ As/GaAs quantum dots. ${ }^{3,4}$ In particular, since the first demonstration of $1.3 \mu \mathrm{m}$ emission at room temperature from InAs/GaAs short period superlattices (SPS), ${ }^{5}$ several groups have reported $1.3 \mu \mathrm{m}$ emission from ordered $\operatorname{In}(\mathrm{Ga})$ As alloys or short period $(\mathrm{InAs})_{m} /(\mathrm{GaAs})_{n}$ superlattices, (where $m, n$ can be $<1$ ) forming digital alloys. ${ }^{3,6}$ There have also been reports of $\sim 1.3 \mu \mathrm{m}$ emission from InAs quantum dots buried in an InGaAs well ${ }^{7}$ or covered with an InGaAs or InAlAs layer. ${ }^{8}$ Room-temperature lasing at $1.3 \mu \mathrm{m}$ and resonant cavity photodiodes, ${ }^{9,10}$ using such heterostructures have also been demonstrated.

Although the SPS growth techniques for quantum dots are seemingly similar, there are subtle differences in the manner in which the group III and V components are delivered onto the growing substrate. It has been suggested by Mukai et al. that these dots may be formed due to "compositional nonuniformities during two dimensional growth," rather than by the conventional strain driven StranskiKrastanow (S-K) growth. ${ }^{4}$ It is also possible that formation of an alloy and energy minimization under strain conditions, together with adatom kinetics and In-Ga interdiffusion leads to the formation of these dots. ${ }^{11}$ While detailed structural

\footnotetext{
${ }^{a)}$ Electronic mail: pkb@eecs.umich.edu
}

characterization of quantum dots grown by conventional self-organized growth has been reported, there is no reported data, to our knowledge, from transmission electron microscopy (TEM) of superlattice dots. In this letter, we report on the TEM characterization of quantum dots formed by cycled submonolayer deposition (CSD) of InAs and GaAs grown on GaAs substrates. This is compared with the characteristics of similar dots formed by conventional $(\mathrm{S}-\mathrm{K})$ growth, with solid source molecular beam epitaxy (MBE). Additionally, we have performed temperature dependent photoluminescence measurements to elucidate the presence and the role of defects.

\section{EXPERIMENTAL DETAILS}

The experimental heterostructures were grown in Varian Gen II solid source $\left(\mathrm{Ga}, \mathrm{In}, \mathrm{As}_{4}\right) \mathrm{MBE}$ systems. The first set of CSD samples were grown as follows. After the growth of a $0.5 \mu \mathrm{m} \mathrm{GaAs}$ buffer layer on (001) semi-insulating GaAs at $600{ }^{\circ} \mathrm{C}$, the growth temperature was ramped down to $510^{\circ} \mathrm{C}$. Fractional monolayers (ML) of InAs $(m)$ and GaAs (n) ( $m$ and $n$ varying from 0.25 to 0.8 ) were then grown with a $5 \mathrm{~s}$ pause between the layers. The $\mathrm{As}_{4}$ shutter was kept open during the pause. This cycle was then repeated several times. The substrate temperature was then raised to $600{ }^{\circ} \mathrm{C}$ and a $0.3 \mu \mathrm{m} \mathrm{GaAs}$ layer was grown. The entire heterostructure is undoped. We will refer to these samples as CSD1. Another batch of heterostructures with buried CSD quantum dots were grown slightly differently, as follows. The growth temperature for this heterostructure was $610^{\circ} \mathrm{C}$ except for the quantum dot (QD) region. Epitaxial growth was initiated with a $1.5 \mu \mathrm{m}$ GaAs buffer layer, followed by a $900 \AA$ GaAs/AlGaAs SPS with average composition of $\mathrm{Al}_{0.15} \mathrm{Ga}_{0.85} \mathrm{As}$, a $400 \AA \mathrm{AaAs}$ barrier with the QDs in the GaAs center, a $300 \AA$ GaAs/AlGaAs short period superlat- 


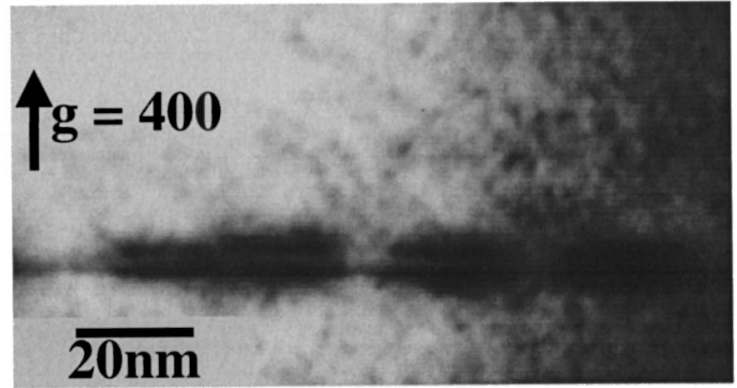

(a)

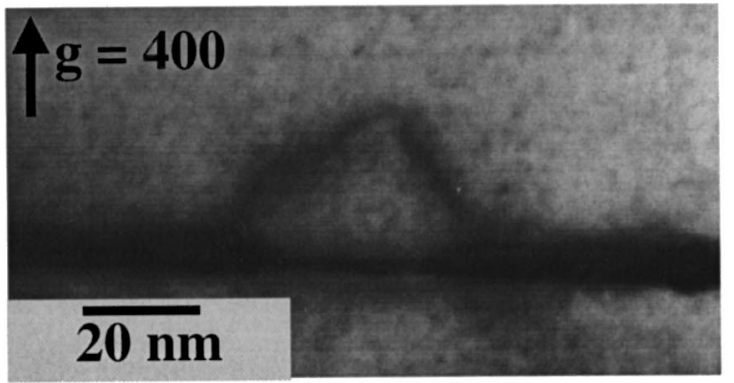

(b)

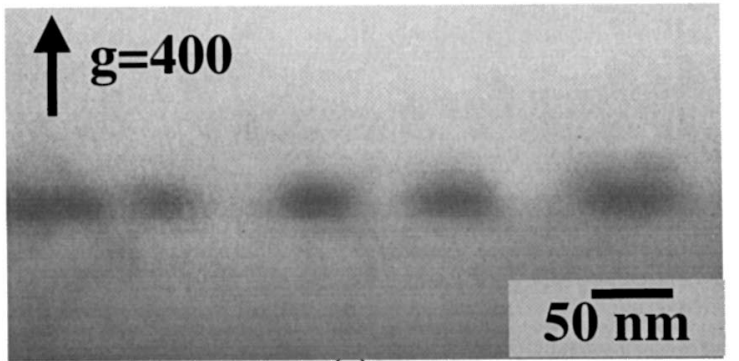

(c)

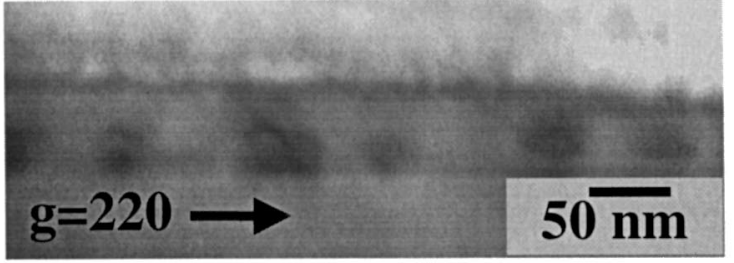

(d)

FIG. 1. XTEM images of quantum dots grown by cycled submonolayer deposition (CSD) on (100) GaAs substrate: (a) 7 periods 0.5 ML InAs/0.5 ML GaAs, (b) 10 periods $0.5 \mathrm{ML}$ InAs/0.5 ML GaAs, (c) 20 periods 0.25 ML InAs/0.25 ML GaAs, (d) 22 periods of $0.25 \mathrm{ML}$ In/0.25 ML Ga/5 As. (a), (b), and (c) are CSD1 dots and (d) is CSD2 dot sample.

tice $\left(\mathrm{Al}_{0.15} \mathrm{Ga}_{0.85} \mathrm{As}\right)$ and a GaAs cap layer. The QDs were grown at $510{ }^{\circ} \mathrm{C}$ by cycled submonolayer depositions of 0.25 monolayers of In and 0.25 monolayers of Ga separated by $5 \mathrm{~s}$ pauses under As flux. The QDs were formed from a total deposition of 11 monolayers, which were then covered by an additional $100 \AA$ of GaAs before increasing the substrate temperature back to $610^{\circ} \mathrm{C}$. We will refer to these samples as CSD2. For comparison purposes, $\mathrm{In}_{0.4} \mathrm{Ga}_{0.6}$ As and InAs self-organized quantum dots were also grown by the conventional technique, the details of which have been described elsewhere. ${ }^{12}$ Very briefly, approximately $2 \mathrm{ML}$ of InAs or InGaAs were deposited after the transformation of the in situ reflective high energy electron diffraction (RHEED) pattern from a streaky to a spotty one. We will refer to these as

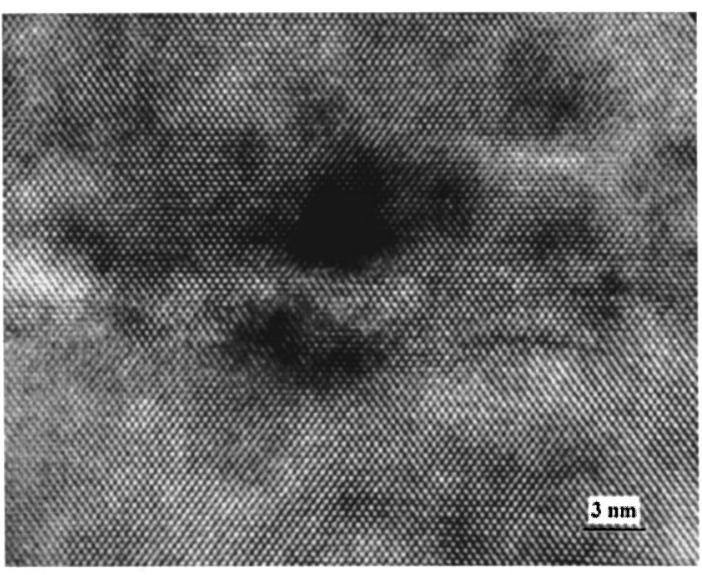

FIG. 2. Lattice image of a single quantum dot grown by CSD of 7 periods of $0.5 \mathrm{ML}$ InAs/0.5 ML GaAs. The dark region in the center suggests an In rich core.

conventional samples. The top GaAs overlayer is not grown in samples intended for atomic force microscopy (AFM) measurements.

\section{RESULTS AND DISCUSSIONS}

Structural characterization of the dots was done by AFM and transmission electron microscopy (TEM) measurements. From the former, the dot densities in the conventional and the CSD samples was found to be $\sim 10^{11}$ and $10^{10} \mathrm{~cm}^{-2}$, respectively. Figures 1(a) and 1(b) show the cross-sectional TEM (XTEM) micrographs of CSD1 samples grown with 7 and 10 periods, respectively, of 0.5 ML InAs/0.5 ML GaAs to form the quantum dots. These images indicate the presence of a wetting layer in this dot system. Figure 1(c) depicts the XTEM micrograph of a sample in which the quantum dot is formed by 20 periods of $0.25 \mathrm{ML}$ InAs/0.25 ML GaAs. No dislocations are observed in the 7 period $(0.5 \mathrm{ML} / 0.5 \mathrm{ML})$ sample, but dislocations begin to appear in the 10 period sample. On the other hand, the dots formed with 20 periods of $0.25 \mathrm{ML} / 0.25 \mathrm{ML}$ are apparently defect free. In fact, dislocations were not observed in samples in which the total InAs thickness was 5-6 ML or less, whereas a network of threading and misfit dislocations are visible in samples in which the InAs thickness exceeds 6 ML. Similar data for the CSD2 sample is shown in Fig. 1(d). Again, it is observed that no dislocations are formed when the total InAs thickness is $6 \mathrm{ML}$ or less.

TABLE I. Comparison of structural parameters and luminescence characteristics of cycled submonolayer deposition dots with those of conventional quantum dots.

\begin{tabular}{ccc}
\hline \hline $\begin{array}{c}\text { Quantum dot } \\
\text { parameter }\end{array}$ & Conventional dots & $\begin{array}{c}\text { Cycled submonolayer } \\
\text { dots }\end{array}$ \\
\hline Width & $20 \mathrm{~nm}$ & $29 \mathrm{~nm}$ \\
Height & $7 \mathrm{~nm}$ & $11 \mathrm{~nm}$ \\
Density & $\sim 10^{11} \mathrm{~cm}^{-2}$ & $\sim 10^{10} \mathrm{~cm}^{-2}$ \\
PL emission peak at & $1.0 \mu \mathrm{m}$ & $1.3 \mu \mathrm{m}$ \\
$T=300 \mathrm{~K}(e 1-h h 1)$ & & \\
\hline \hline
\end{tabular}




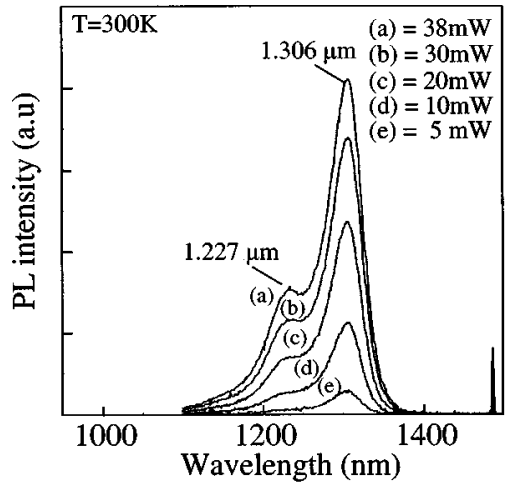

(a)

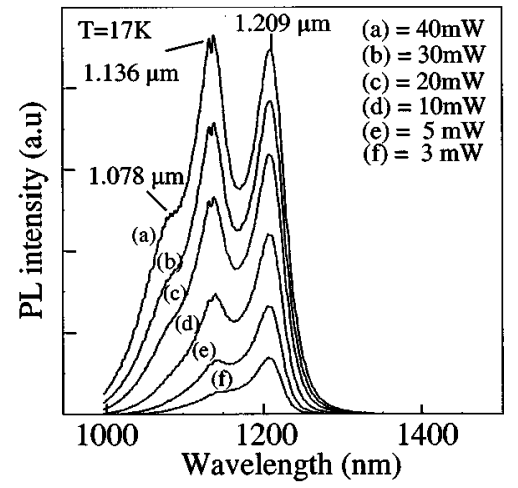

(b)

FIG. 3. (a) Room-temperature and (b) low temperature photoluminescence from a CSD1 sample with 16 periods of $0.25 \mathrm{ML}$ InAs/0.25 ML GaAs dots.

A high resolution XTEM micrograph of a single quantum dot in a CSD1 sample with 7 periods of $0.5 \mathrm{ML}$ InAs/ $0.5 \mathrm{ML}$ of GaAs, observed with (100) reflection, is shown in Fig. 2. It is apparent that the dot is more disk-like, than oval or pyramidal, in shape. It is also evident that the core of dot is In rich and may even be InAs, while the outer periphery may be GaAs rich, as no lattice-constant change is observable at the boundaries. An indium rich core has been predicted by thermodynamic calculations. ${ }^{13}$ This is in agreement with the observations of Mukai et al., ${ }^{4}$ who also observed distinct dark spheres within the short-period layers in their TEM micrograph. This led them to believe that these dots were not formed by $\mathrm{S}-\mathrm{K}$ growth, although the TEM images indicated the presence of a wetting layer. From our TEM and AFM studies, the structural parameters of the CSD dots are obtained and are compared with the conventional dots in Table I. The CSD dot dimensions are found to be about 1.5 times the conventional dots, in accordance with an earlier report. $^{8}$

Photoluminescence spectra of the quantum dot samples were measured at different temperatures from $17 \mathrm{~K}$ to room temperature. Photoluminescence data at 300 and $17 \mathrm{~K}$ from a CSD1 sample with 16 periods of $0.25 \mathrm{ML}$ InAs/0.25 ML GaAs are shown in Fig. 3. Peaks and shoulders corresponding to ground and excited state transitions, respectively, are observed in the spectra. The peak corresponding to the ground state transition occurs at $1.3 \mu \mathrm{m}$. The energy separation between the ground and first excited state transition peaks is approximately $62 \mathrm{meV}$ at room temperature and 66 $\mathrm{meV}$ at $17 \mathrm{~K}$ and the separation between the first and second

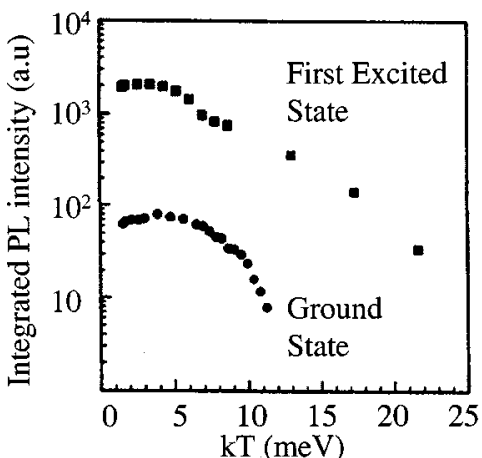

(a)

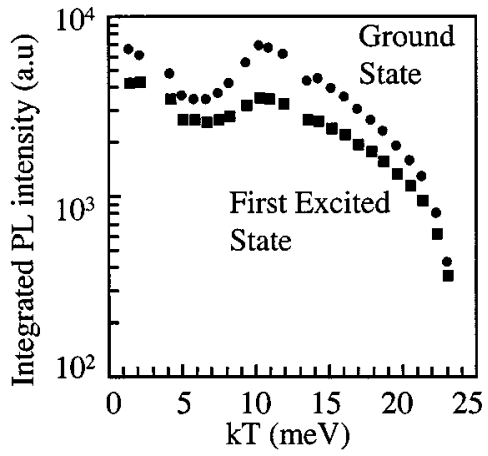

(b)

FIG. 4. (a) Wavelength integrated intensity of the ground state and the excited state transition as a function of the temperature for dots grown by the conventional technique; (b) similar data for the CSD dots grown with 20 periods of $0.25 \mathrm{ML}$ InAs/0.25 ML GaAs.

excited states at $17 \mathrm{~K}$ is $\sim 59 \mathrm{meV}$. These energy separations are in excellent agreement with electroluminescence data published earlier. ${ }^{6}$ The luminescence peak from the conventional sample occurs at about $1 \mu \mathrm{m}$. Temperature dependence of the PL intensity from the conventional dot sample is shown in Fig. 4(a). The wavelength-integrated intensity remains constant or increases slightly upto about $75 \mathrm{~K}$ and then decays due to ionization of the localized exciton. ${ }^{12}$ The intensity of the ground-state emission drops off dramatically, whereas the excited state transition first decays at a fast rate, followed by a slow decay till room temperature. The fast decay rate indicates nonradiative transitions associated with a defect level while the slow decay of the excited state reflects normal thermal quenching. It is likely that compositional mixing ${ }^{13}$ and segregation, which accompanies dot formation, is the origin of the observed defects. The temperature dependence of the photoluminescence (PL) intensity of the ground and excited state transitions for a CSD dot sample with 20 periods of $0.25 \mathrm{ML}$ InAs/0.25 ML GaAs is depicted in Fig. 4(b). Again the general behavior is the same as Fig. 4(a), but the role of defects is more prominent. In fact, no slow decay is observed but multiple fast decays with temperature are present. From these measurements it is apparent that although there are no $\left(<10^{4} \mathrm{~cm}^{-2}\right)$ misfit dislocations at or near the dots, there are other nonradiative defects, intrinsic to the formation of the dots, present in them. These defects, caused by segregation, surface compositional mixing, and other nonideal adatom migration effects, are possibly at the island/GaAs boundaries. 


\section{CONCLUSIONS}

In conclusion, the structural and luminescence properties of quantum dots grown by cycled submonolayer on (100) GaAs were studied. The CSD dots were found to be $\sim 1.5$ times larger with an areal density about 10 times lower than conventional dots. TEM images reveal that defects begin to appear in samples in which the overall InAs thickness is greater than 5-6 ML. Strong room temperature luminescence with multiple transition peaks was observed from the CSD samples. The peak energy of the ground state transition is at $1.3 \mu \mathrm{m}$. From the rapid decay of the wavelength integrated PL intensity as a function of temperature, it is apparent that the quantum dots have nonradiative defects, intrinsic to dot formation, present in them.

\section{ACKNOWLEDGMENTS}

The authors acknowledge Dr. K. Kamath for his contributions. The work is being supported by the Army Research Office under the MURI program (Grant No. DAAG55-98-10288).
${ }^{1}$ M. Kondow, K. Uomi, A. Niwa, T. Kitatani, S. Watahiki, and Y. Yazawa, Jpn. J. Appl. Phys., Part 1 35, 1273 (1996).

${ }^{2}$ H. P. Xin and C. W. Tu, Appl. Phys. Lett. 72, 2442 (1998).

${ }^{3}$ R. P. Mirin, J. P. Ibbetson, K. Nishi, A. C. Gossard, and J. E. Bowers, Appl. Phys. Lett. 67, 3795 (1997).

${ }^{4}$ K. Mukai, N. Ohtsuka, M. Sugawara, and S. Yamazaki, Jpn. J. Appl. Phys., Part 2 33, L1710 (1994).

${ }^{5}$ E. Roan and K. Cheng, Appl. Phys. Lett. 59, 2688 (1991).

${ }^{6}$ D. L. Huffaker and D. G. Deppe, Appl. Phys. Lett. 73, 520 (1998).

${ }^{7}$ V. M. Ustinov, N. A. Maleev, A. E. Zhukov, A. R. Kovsh, A. Yu. Egorov, A. V. Lunev, B. V. Volovok, I. L. Krestnikov, Yu. G. Musikhin, N. A. Bert, P. S. Ko'pev, Zh. I. Alferov, Ledentsov, and D. Bimberg, Appl. Phys. Lett. 74, 2815 (1999).

${ }^{8}$ K. Nishi, H. Saito, S. Sugou, and J-S. Lee, Appl. Phys. Lett. 74, 111 (1999).

${ }^{9}$ D. L. Huffaker, G. Park, Z. Zou, O. B. Shchekin, and D. G. Deppe, Appl. Phys. Lett. 73, 2564 (1998).

${ }^{10}$ J. C. Campbell, D. L. Huffaker, H. Deng, and D. G. Deppe, Electron. Lett. 33, 1337 (1997).

${ }^{11}$ I. Mukhametzanov, R. Heitz, J. Zeng, P. Chen, and A. Madhukar, Appl. Phys. Lett. 73, 1841 (1998).

${ }^{12}$ K. Kamath, P. Bhattacharya, and J. Phillips, J. Cryst. Growth 175/176, 720 (1997).

${ }^{13}$ J. Tersoff, Phys. Rev. Lett. 81, 3183 (1998). 